In der Rubrik „Literatur kompakt" dieser Ausgabe referieren und kommentieren diese Experten für Sie Arbeiten aus der internationalen Fachliteratur.

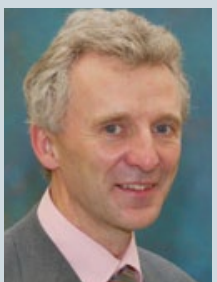

Dr. med. Martin Claßen, Bremen

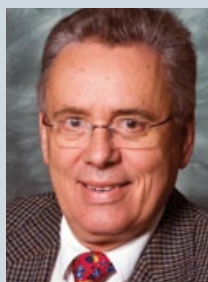

Dr. med. Hartmut Koch, Vechta

\section{Zucker oder Süßstoff im Softdrink - das ist hier die Frage}

Die Tatsache, dass der Genuss süßer Softdrinks für eine exzessive Gewichtszunahme verantwortlich sein kann, ist unstrittig. Deshalb enthalten viele der Getränke Süßstoff. Bislang gab es aber keine guten Daten, die zeigten, dass der Ersatz des Zuckers durch Süßstoff eine Gewichtszunahme wirklich vermindert. Gleich zwei Arbeitsgruppen legen hierzu nun eindeutige Daten vor.

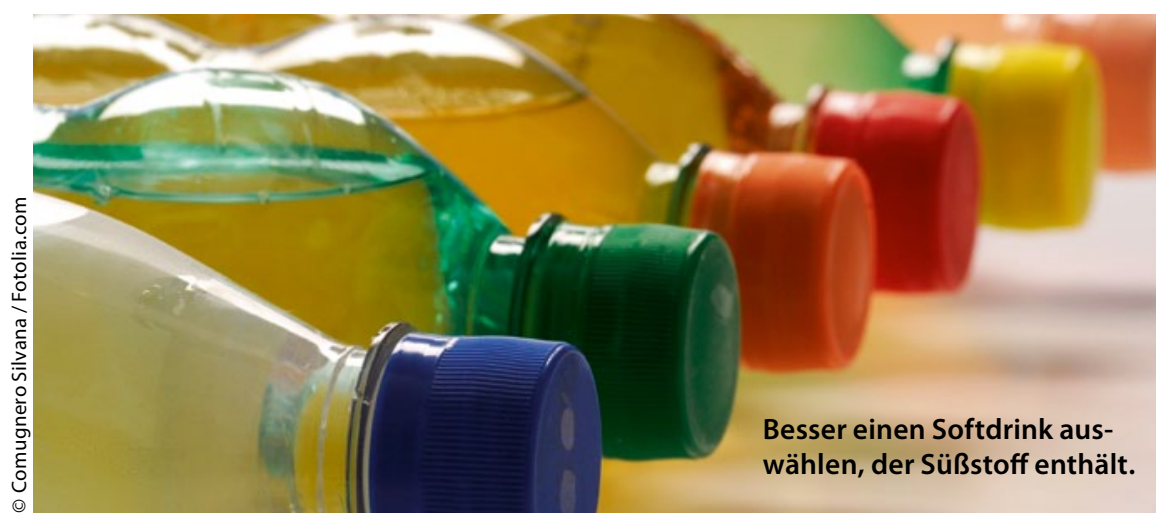

anne de Ruyter und Kollegen aus Amsterdam [1] sowie Cara Ebbeling et al. aus Boston [2] präsentieren jeweils eine prospektive, randomisierte Doppelblind-Studie aus unterschiedlichen pädiatrischen Altersgruppen. Die holländische Studie umfasste 641 normalgewichtige Kinder im Alter von knapp fünf bis knapp zwölf Jahren. Die amerikanische Studie untersuchte dagegen 224 übergewichtige bzw. adipöse Jugendliche.

In die Studie aus den Niederlanden wurden nur Kinder aufgenommen, die auch vor Beginn der Studie schon regelmäßig mit Zucker gesüßte Softdrinks zu sich nahmen. Den Kindern wurde täglich eine 250-ml-Packung eines iden- tisch aussehenden Softdrinks zur Verfügung gestellt. Die eine Gruppe erhielt ein Getränk mit Süßstoff, die zweite Gruppe ein nicht unterscheidbares Getränk mit 26 g Saccharose (104 kcal pro Tag). Außerdem wurden das Körpergewicht, die Größe und die Hautfaltendicke an verschiedenen Stellen gemessen. Die Gewichtszunahme in der Gruppe, die Süßstoff verwendete, betrug innerhalb von 18 Monaten $6,35 \mathrm{~kg}$, in der Gruppe mit dem zuckerhaltigen Getränk dagegen $7,37 \mathrm{~kg}$.

In der amerikanischen Studie von Ebbeling und Mitarbeitern waren die Probanden deutlich älter und zu Beginn der Studie bereits übergewichtig bzw. adipös. Während des ersten Jahres wurden in der Interventionsgruppe kalorienfreie Getränke (Wasser oder mit Süßstoff versehene Softdrinks) nach Hause geliefert. Außerdem erhielten die Probanden dieser Gruppe alle zwei Wochen einen Anruf und wurden alle vier Monate besucht mit dem Ziel, den Verbrauch an zuckerhaltigen Getränken zu vermindern. Verglichen wurden die Jugendlichen mit einer Kontrollgruppe. Die Interventionsdauer betrug zwölf Monate, die Nachbeobachtungszeit weitere zwölf Monate. Nach dem ersten Jahr zeigte sich in der Interventionsgruppe lediglich bei der ethnischen Gruppe der Latinos eine geringere Zunahme des Body-Mass-Index. Dieser Befund konnte am Ende des zweiten Jahres jedoch nicht bestätigt werden.

1. de Ruyter JC et al. A trial of sugar-free or sugar-sweetened beverages and body weight in children. N Engl J Med 2012; 367: 1397-406 2. Ebbeling $C B$ et al. A randomized trial of sugarsweetened beverages and adolescent body weight. N Engl J Med 2012; 367: 1407-16

Kommentar: Die Adipositas ist mittlerweile eine der größten globalen Gesundheitsherausforderungen des 21 . Jahrhunderts. Die Untersuchungen zeigen, dass eine sinnvolle Intervention beginnen muss, bevor die Kinder übergewichtig sind. In einem Editorial weist Sonia Caprio [N Engl J Med 2012; 367: 1462-3] darauf hin, dass sich auf den Stoffwechsel besonders die billigen, stark fruktosehaltigen Süßungsmittel aus hydrolisierter Maisstärke negativ auswirken. Sie fordert institutionelle Interventionen durch die zuständigen Fachgesellschaften und Behörden. Sie erwähnt nicht den ausgeprägten Lobbyismus der weltweiten $\mathrm{Zu}$ ckerindustrie. Das sinnvolle Verbot zuckerhaltiger Softdrinks wird wohl noch auf sich warten lassen.

Dr. Hartmut Koch 